\title{
THE SIXTEENTH ANNUAL MEETING OF THE AMERICAN MATHEMATICAL SOCIETY.
}

THE sixteenth annual meeting of the Society was held at Boston on Tuesday, Wednesday, and Thursday, December 2830,1909 , in affiliation with the American association for the advancement of science. Tuesday afternoon was devoted to a joint session with Sections $\mathrm{A}$ and $\mathrm{B}$ of the Association. A joint session was held with section A on Wednesday morning, the programme consisting of Professor Keyser's vice-presidential address "On the thesis of modern logistic," a report by Professor D. E. Smith on "The work of the International Commission on the teaching of mathematics," and the papers numbered (1) and (2) in the list below. Separate sessions of the Society were held on Wednesday afternoon and on Thursday morning and afternoon. On Tuesday evening several members took advantage of an invitation to attend the dinner and smoker of the Association of mathematical teachers in New England. The annual dinner of the Society took place on Wednesday evening, forty-seven members gathering for this agreeable occasion. Much credit for the success of the meeting must be given to the local committee on arrangements, Professors Tyler, Bartlett, and Bouton.

The total attendance at the annual meeting included the following sixty-one members :

Dr. C. S. Atchison, Professor D. P. Bartlett, Dr. E. G. Bill, Professor G. D. Birkhoff, Professor C. L. Bouton, Professor E. W. Brown, Dr. J. E. Clarke, Mr. G. R. Clements, Professor F. N. Cole, Professor L. L. Conant, Professor J. L. Coolidge, Mr. C. H. Currier, Mr. F. F. Decker, Dr. F. J. Dohmen, President E. A. Engler, Professor T. C. Esty, Professor F. C. Ferry, Professor W. B. Fite, Professor W. A. Garrison, Miss A. B. Gould, Professor F. L. Griffin, Professor J. N. Hart, Professor C. N. Haskins, Professor L. A. Howland, Professor E. V. Huntington, Dr. L. C. Karpinski, Professor O. D. Kellogg, Professor C. J. Keyser, Professor Gaetano Lanza, Dr. D. D. Leib, Dr. N. J. Lennes, Mr. Joseph Lipke, Dr. J. V. McKelvey, Professor H. P. Manning, Professor W. H. Metzler, 
Professor G. A. Miller, Professor J. A. Miller, Mr. H. H. Mitchell, Professor W. A. Moody, Professor C. L. E. Moore, Professor G. D. Olds, Dr. F. W. Owens, Dr. H. B. Phillips, Dr. Arthur Ranum, Professor R. G. D. Richardson, Mr. W. J. Risley, Professor E. D. Roe, Professor Mary E. Sinclair, Professor D. E. Smith, Miss M. E. Trueblood, Professor H. W. Tyler, Professor J. M. Van Vleck, Professor Oswald Veblen, Professor H. S. White, Professor J. K. Whittemore, Professor D. T. Wilson, Professor E. B. Wilson, Dr. Ruth G. Wood, Professor T. W. D. Worthen, Mr. W. C. Wright, Professor Alexander Ziwet.

Ex-President H. S. White and Professor E. W. Brown occupied the chair alternately during the several sessions. The Council announced the election of the following persons to membership in the Society: Professor R. M. Barton, Dartmouth College ; Dr. J. R. Conner, Johns Hopkins University ; Miss Eva M. Smith, London, England. Nine applications for membership were received.

The reports of the Treasurer, Auditing Committee, and Librarian have recently appeared in the Annual Register. The membership of the Society has increased during the year from 601 to 618 , including at present 58 life members. The number of papers presented at all meetings during the year 1909 was 149 . The total attendance of members at the meettings was 311 . The Treasurer's report shows a balance of $\$ 8003.78$, of which $\$ 3581.70$ is credited to the life-membership fund.* Sales of the Society's publications during the year amounted to $\$ 1748.90$. The Library has increased to nearly 3300 volumes. A catalogue of the Library, corrected to January 1, 1910, has been issued as a separate publication.

At the annual election, which closed on Thursday morning, the following officers and other members of the Council were chosen :

$$
\begin{array}{ll}
\text { Vice-Presidents, } & \begin{array}{l}
\text { Professor L. E. Dickson, } \\
\text { Professor J. I. Hutchinson. }
\end{array} \\
\text { Secretary, } & \text { Professor F. N. CoLE. }
\end{array}
$$

\footnotetext{
* The total income of the Society since January 1,1895 , has been $\$ 56,299.22$, of which $\$ 30,310.94$ is credited to members' dues. Disbursements in the same period have been $\$ 49,412.11$, of which $\$ 39,008.47$ has been expended for printing the Bulletin, Transactions, and other publications of the Society. The total returns from publications have been $\$ 13,508.99$. Editorial expenses have been $\$ 2750.15$, administrative expenses $\$ 7653.14$.
} 


$\begin{array}{ll}\text { Treasurer, } & \text { Professor J. H. TANneR. } \\ \text { Librarian, } & \text { Professor D. E. SмiтH. }\end{array}$

Committee of Publication,

Professor F. N. Cole,

Professor E. W. BRown,

Professor Virgil SNyder.

Members of the Council to serve until December, 1912,

Professor D. R. Curtiss, Professor J. C. Fields,

Professor L. P. Eisenhart, Professor P. F. Smith.

The following papers were read at this meeting :

(1) Professor F. L. Griffin : "Certain tests comparing areas and other geometrical magnitudes."

(2) Professor G. A. Miller : "Groups generated by two operators $s_{1}, s_{2}$ satisfying the equation $s_{1} s_{2}^{2}=s_{2} s_{1}^{2}$."

(3) Dr. H. M. ShefFer : "Total determinations of deductive systems with special reference to the algebra of logic."

(4) Professor R. G. D. Richardoson : "The Jacobi criterion in the calculus of variations and the oscillation of solutions of $m$ linear differential equations of the second order with $m$ parameters."

(5) Dr. J. V. McKelvey : "The groups of birational transformations of algebraic curves of genus five."

(6) Professor J. L. Coolidge : "The representation by means of circles of the imaginary elements of a three-dimensional domain."

(7) Dr. L. C. Karpinski : “Jordanus Nemorarius and John of Halifax."

(8) Mr. H. H. Mitchell : "The subgroups of the collineation group of the finite plane, $P G(2, p) . "$

(9) Professor W. H. JACKsON : "Differential and integral equations arising out of the theory of radiation."

(10) Professor G. D. Brrkhoff : "The stable solutions of the problem of three bodies."

(11) Professor W. D. CaIrns: "The solution of the Lagrange equation in the calculus of variations by means of integral equations."

(12) Dr. Arthur Ranum: "On the line geometry of r emannian space." 
(13) Dr. H. F. MAcNeish : "Linear polars of quantics which are completely reducible to the product of linear forms."

(14) Professor E. V. Huntington : "An elementary explanation of the precession of a gyroscope."

(15) Professor C. J. KeYser : "Relational groups."

(16) Professor EDward Kasner: "Thomson and Tait's theorem on conservative forces."

(17) Professor Edward Kasner : “Note on Lamés families connected with dynamics."

(18) Dr. Arthur Ranum : "On Clifford parallels and Clifford surfaces in riemannian space."

Dr. Sheffer was introduced by Professor Osgood. In the absence of the authors, Dr. MacNeish's paper was read by Professor Veblen, and the papers of Professors Jackson, Cairns, Keyser, and Kasner were read by title. Abstracts of the papers follow below. The abstracts are numbered to correspond to the titles in the list above.

1. In this note Professor Griffin observes that the process used in an earlier paper* for comparing areas, lengths, etc., of central orbits, can be applied to the purely geometrical problem of making similar tests in many cases where two curves are given by either polar or rectangular equations or by differential equations of the first or second order.

2. In 1878 Cayley published a note on the groups which may be generated by two operators $s_{1}, s_{2}$ satisfying the equation $s_{1} s_{2}=s_{2}^{2} s_{1}^{2}$ and observed that it is not possible to represent all the operators of such a group in the form $s_{1}^{a} s_{2}^{\beta}$ except when the group is cyclic. In 1905 Netto considered the same relation in Crelle and observed that either the orders of $s_{1}, s_{2}$ are equal to each other or the order of one of these operators is twice the order of the other. In a recent number of the Quarterly Journal Professor Miller extended these results, but did not give many general theorems. In the present paper he gives several fundamental theorems which are implied in the above relation, and by means of these he obtains the known results much more easily and also arrives at a number of new results. In particular he shows that either the first or second of the three generational relations $s_{1}^{5}=s_{2}^{5}=1, s_{1} s_{2}^{2}=s_{2} s_{1}^{2}$ given by

\footnotetext{
* Read December 30, 1908.
} 
Netto is redundant. That is, the two conditions $s_{1}^{5}=1$, $s_{1} s_{2}^{2}=s_{2} s_{1}^{2}$ imply that $s_{2}^{5}=1$.

The following are some of the theorems established by Professor Miller in the present article: If two operators satisfy the relation $s_{1} s_{2}^{2}=s_{2} s_{1}^{2}$, their squares are of the same order as their product, and they generate a group whose commutator subgroup is generated by two conjugate commutators and whose commutator quotient group is cyclic. If both of these operators are of odd order, they generate a solvable group whose commutator subgroup is either cyclic or the direct product of two cyclic groups. If $\alpha$ belongs to exponent 7 with respect to a prime number $p$ and if $\alpha+1 \equiv \alpha^{2} \bmod p$, then $p=29$ and $\alpha=24$. Similar characteristic properties are established for the primes 5, 11, 19 as incidental results. It is also proved that $\left(s_{1} s_{2}^{-1}\right)^{4 n}=s_{2}^{-1}\left(s_{1}^{6} s_{2}^{-6}\right) s_{2}$ whenever $s_{1}, s_{2}$ are both of odd order.

3. Basal determinations ("postulate definitions") of various deductive systems - for example, of "ordinary" algebra, of the "algebra of logic," and of geometry - in terms of conveniently chosen (or basal) operations or relations have been developed recently. The present paper discusses the problem of the total determinations of deductive systems, that is, the total sets of element classes, relations, and propositions that are possible for a given system. The paper is thus intended as an introduction to a general theory of systems.

In particular, Dr. Sheffer solves the problem for the deductive system called the algebra of logic. He finds the total set of relations (and operations) which can serve as basal relations (and operations) for that algebra. Of this total set, the relations and operations employed hitherto - " logical addition," "logical multiplication," and "inclusion" (Huntington); the "between"-relation (Kempe); and the O-relation (Royce) - are shown to be isolated cases.

4. The relation between the Jacobi criterion of the calculus of variations and the oscillation of the solution of the self-adjoint differential equation of the second $\operatorname{order}(p u)+q u+\lambda k u=0$ was the subject of a paper read by Professor Richardson at the summer meeting of the Society. In a second paper of a series to appear in the Mathematische Annalen the results have been 
extended to the case of $m$ linear differential equations

$$
\left(p_{i} u_{i}^{\prime}\right)^{\prime}+q_{i} u_{i}+\left(\lambda r_{i 1}(x)+\cdots+\pi r_{i n}(x)\right) u_{i}=0 \quad(i=1,2, \cdots, m)
$$

with the $m$ parameters $\lambda, \cdots, \pi$. These differential equations are considered as the Lagrange equations of a calculus of variations problem, the functions $u_{i}(x)$ satisfying the boundary conditions $u_{i}(0)=u_{i}(1)=0$ and a certain quadratic condition or the quadratic and $\mathrm{nm}$ linear conditions. The Jacobi criterion in each case determines exactly the number $n$ of oscillations of the function $u_{i}(x)$ in the interval 0,1 .

5. The purpose of Dr. McKelvey's paper was to find the normal curves of hyperspace of genus 5 and the forms of the plane curves into which they can be projected; also to find the groups of birational transformations under which they are invariant. The plane curves of this genus are in general sextics with 5 double points. They become nodal quintics when a $g_{3}^{1}$ exists. The equations of the sextics were obtained by means of the quadratic relations among their adjoint curves of order 3. The curve of genus 5 is the only one which is completely defined by the quadratic relations among its adjoints of order $n-3$. It was shown that the transformations of the sextic are related to the linear transformations of a certain plane quintic, except when the quadratic relations among the adjoints are also invariant. The largest group obtained was of order 192.

6. In 1872 Laguerre showed how the imaginary points of the finite three-dimensional domain could be represented by means of real circles of positive or negative radius. Professor Coolidge's paper exhibited two other methods of circular representation which afford a better separation between conjugate imaginaries, and a simpler representation of the simplest point systems.

7. The introduction into Europe of the Hindu methods in arithmetic is closely associated by Moritz Cantor in his Geschichte der Mathematik with the names of Leonard of Pisa and Jordanus Nemorarius. The prominent place given by Cantor to Jordanus rightfully belongs to John of Halifax (Sacrobosco). Dr. Karpinski's paper presents some new material in regard to the Algorismus by Jordanus and some notes on copies 
found in American libraries of the Algorismus by Sacrobosco, and emphasizes the important rôle played by John of Halifax in spreading the system of the Hindus, taught by the Arabs.

8. In Mr. Mitchell's paper the determination is made of the subgroups of the collineation group in three variables, when the coefficients of the transformations $\rho x_{i}^{\prime}=\Sigma a_{i j} x_{j}(i, j=1,2,3)$ are integers reduced modulo $p, p$ being a prime. The order of the group is $\left(p^{2}+p+1\right)\left(p^{2}+p\right) p^{2}(p-1)^{2}$.

The problem is treated geometrically, the $p^{2}+p+1$ sets of marks $(\alpha \beta \gamma)$, upon which the group may be represented as a permutation group, being regarded as the points of a finite plane. A self-conjugate subgroup consisting of all transformations with determinant unity exists if $p$ has the form $3 n+1$. There are subgroups leaving fixed a point, a line, a triangle, and a conic. The only additional subgroups are the $G_{360}$, the Hessian $G_{216}$, the $G_{168}$, and two subgroups of the Hessian, $G_{72}$ and $G_{36}$. The methods employed in the determination of the groups of the latter class apply equally well to the case of finite groups in the ordinary geometry.

9. Schuster has discussed the transmission of radiant heat when the isothermal surfaces are parallel planes and conduction is neglected. The problem was reduced by him to the solution of the following equation :

$$
\left(1+\frac{\partial}{\partial t}\right) \frac{\partial^{2} E}{\partial \mu^{2}}=\frac{\partial E}{\partial t}
$$

where $E$ is the total density of radiation at any point. The aim of the discussion was to describe only the broad features of the phenomena and the following assumptions were made: (i) $E$ is independent of the time, (ii) the radiation flows only normally to the isothermal planes, (iii) the absorption is independent of the wave length.

In the present paper, Professor Jackson finds that the removal of restriction (i) gives the length of time necessary to establish the steady state to a given degree of approximation, while the removal of restrictions (ii) and (iii) leads respectively to the following equations, which are reducible to ordinary integral equations of the second kind with symmetric kernel :

$$
\left(1+\frac{\partial}{\partial t}\right)\left(1-x^{2} \frac{\partial^{2}}{\partial \mu^{2}}\right) E=\int_{0}^{1} E d x
$$


where $t, x, \mu$ are independent variables,

$$
\left(1+\frac{\partial}{\partial t}\right)\left(1-f(\kappa) \frac{\partial^{2}}{\partial m^{2}}\right) E=\int_{0}^{1} E d \kappa
$$

where $t, \kappa, m$ are independent variables.

10. Professor Birkhoff discusses the stable solutions of the problem of three bodies in the light of an earlier paper on stability presented by him at the Princeton meeting, September 14,1909 .

11. The method of integral equations is applied by Professor Cairns to the Lagrange problem : To determine $y_{1}, \cdots, y_{n}$ as functions of $x$ so that they make

$$
I=\int_{a}^{b} F\left(y_{1}^{\prime}, \cdots, y_{n}^{\prime} ; y_{1}, \ldots, y_{n} ; x\right) d x
$$

a minimum and at the same time satisfy given differential equations

$$
\phi_{i}\left(y_{1}^{\prime}, \ldots, y_{n}^{\prime} ; y_{1}, \ldots, y_{n} ; x\right)=0 \quad(i=1,2, \ldots, m<n) .
$$

(This includes isoperimetric problems as a special case.)

The requirement that the second variation

$$
\int_{a}^{b} Q d x
$$

shall be positive, calls for the existence of functions satisfying the equations (given for simplicity's sake for $n=2$ )

$$
\begin{gathered}
L_{1}(u)+\lambda k_{1} u_{1}+\lambda^{\prime}\left(\frac{\partial \phi}{\partial y_{1}}-\frac{d}{d x} \frac{\partial \phi}{\partial y_{1}^{\prime}}\right)=0, \\
L_{2}(u)+\lambda k_{2} u_{2}+\lambda^{\prime}\left(\frac{\partial \phi}{\partial y_{2}}-\frac{d}{d x} \frac{\partial \phi}{\partial y_{2}^{\prime}}\right)=0, \\
u_{1}^{\prime} \frac{\partial \phi}{\partial y_{1}^{\prime}}+u_{2}^{\prime} \frac{\partial \phi}{\partial y_{2}^{\prime}}+u_{1} \frac{\partial \phi}{\partial y_{1}}+u_{2} \frac{\partial \phi}{\partial y_{2}}=0,
\end{gathered}
$$


where

$$
L_{i}(u) \equiv \frac{1}{2}\left(\frac{d}{d x} \frac{\partial Q}{\partial u_{i}^{\prime}}-\frac{\partial Q}{\partial u_{i}}\right) .
$$

A system $H_{i j}$ of generalized Green's functions shows this set of equations to be equivalent to the system of integral equations

$$
\begin{gathered}
\phi_{1}(x)=\lambda \int_{a}^{b}\left\{H_{11}(x, \xi) \phi_{1}(\xi)+H_{12}(x, \xi) \phi_{2}(\xi)\right\} d \xi+x_{1}^{\prime} g_{1}(x), \\
\phi_{2}(x)=\lambda \int_{a}^{b}\left\{H_{21}(x, \xi) \phi_{1}(\xi)+H_{22}(x, \xi) \phi_{2}(\xi)\right\} d \xi+x_{2}^{\prime} g_{2}(x), \\
\lambda^{\prime} \int_{a}^{b} \Phi(x) d x+\lambda \int_{a}^{b} \Psi\left(\phi_{1}(x), \phi_{2}(x)\right) d x=0,
\end{gathered}
$$

$g_{i}, \Phi$, and $\Psi$ being known functions.

Finally, the solution of these is reduced by a system of orthogonal functions

$$
\begin{gathered}
\psi_{11}(x), \quad \psi_{12}(x), \quad \ldots ; \quad \psi_{21}(x), \quad \psi_{22}(x), \quad \ldots ; \\
\int_{a}^{b}\left(\psi_{1 i}^{2}+\psi_{2 i}^{2}\right) d x=1, \quad \int_{a}^{b}\left(\psi_{1 i} \psi_{1 j}+\psi_{2 i} \psi_{2 j}\right) d x=0 \quad(i \neq j)
\end{gathered}
$$

to a generalized system of linear equations in an infinite number of variables already developed by the writer.

The theory is completely developed, with a full solution of the original minimum problem.

12. In this paper Dr. Ranum gives a classification of linear complexes, of congruences of order one and class one, and of quadric surfaces having real generators, in riemannian space. He also studies in detail three special classes of quadric surfaces, namely Clifford surfaces, surfaces of revolution, and normal surfaces (those having one generator of each set perpendicular to every generator of the other set).

13. By projective methods Dr. MacNeish obtains a recursion sequence of geometric constructions for the linear polar of a point as to a linear $k$-ad of points, as to a $k$-line in the plane, and in general as to a $k$-hedron in $n$-space. Then, using symbolic 
notation, he shows analytically that the linear polars, obtained synthetically above, harmonize with the analytic polar theory for the $n$-ary $k$-ic which is the product of linear factors. A simple application of these results gives a construction for the linear polar of algebraic curves, surfaces, and spreads. A second application is the consideration of certain configurations from the standpoint of linear polarity. The quadrangle-quadrilateral configuration in the plane is generalized and a self dual configuration in $n$-space is obtained consisting of an $(n+2)$-point and an $(n+2)$-hedron. A second generalization gives an associated $k$-point and $k$-hedron in $n$-space which is reciprocal only for $k=n+2$. The invariantive conditions for reciprocity in the case of 4 collinear points is then considered and some interesting new geometric interpretations of the concomitants of the binary quartic form are obtained.

14. Professor Huntington's paper on the gyroscope obtains by elementary methods the actual accelerations of the several particles of the rotating disc when the axle of the disc is "precessing" with angular velocity $\psi^{\prime}$, and hence provides a direct elementary proof of the well-known fact that the applied forces required to maintain this precession constitute a couple lying in a plane perpendicular to the plane of precession and having a moment equal to $C \omega \psi^{\prime}$, where $C$ is the moment of inertia of the disc about its axle and $\omega$ the angular velocity of spinning. The following rule for the direction of precession is believed to be new : If the applied force be thought of as due to the pressure of a shelf against the axle, the precession will take place in the direction in which the axle would tend to roll along the shelf. The paper also calls attention to an error in a recent text-book, in regard to the motion induced by the applied couple in case precession is prevented. As a matter of fact, if the gyroscope is not allowed to precess, the rotation caused by the applied couple will be exactly the same as if the disc were not spinning.

15. From the postulates (as those of Russell, "Theorie générale des relations," Revue de Mathématiques, volume 7, page 115) of the calculus of relations, it readily follows that relations constitute an infinite class of potence equal at least to that of the continuum. Of the infinitude of classes of relations there is a large finite number of classes that by virtue of their 
comprehensiveness and obviousness may be characterized as fundamental. Such are, for example, the classes known as symmetric, asymmetric, non-symmetric, transitive, intransitive, non-transitive, multiform, uniform, biuniform, and so on, together with the classes resulting from logical addition and multiplication of given fundamental relational classes. Professor Keyser's paper is a preliminary report upon the problem of determining which of the fundamental classes of relations are groups under such rules of combination (logical addition and logical multiplication, for example) as are applicable to relations without exception. Of several scores of relational classes thus examined, it is found that a goodly percentage possess the group property under one or both of the rules mentioned. The paper with detailed results will be published at a later date.

16. If particles are projected from an arbitrary point in all directions with a given speed and acted upon by any field of force, a doubly infinite system of trajectories will be obtained. Professor Kasner shows that only in the conservative case will these curves admit orthogonal surfaces. A second peculiarity of conservative fields is the mutual orthogonality of the three circles of curvature having four-point contact. The main part of the paper relates to a question suggested by the general theorem of Thomson and Tait: With what speeds must particles be projected normally from an arbitrary surface, so that the trajectories described shall form a normal congruence? In general the only solution is that for which the sum of the potential and kinetic energies is constant. For certain exceptional surfaces however other laws are possible.

17. In his second paper Professor Kasner determines those conservative forces for which every system of surfaces of equal action (in the sense of Thomson and Tait) constitutes a family of Lamé, that is, part of a triply orthogonal system. The trajectories are found to be circles. The result may be stated quite simply in optical terms and may be connected with non-euclidean geometry.

18. It is well known that the geometry of a congruence of Clifford parallels corresponds to the point geometry of a sphere. Some of the consequences that Dr. Ranum draws from this 
correspondence are the following. If the distances between three Clifford parallels are $a, b, c$, and if the flux-angles between the three rectangular Clifford surfaces joining them are $a, \beta, \gamma$, then these six quantities are related to one another exactly as if their doubles were the sides and angles of a spherical triangle; e. g., $\sin 2 a: \sin 2 b: \sin 2 c=\sin 2 a: \sin 2 \beta: \sin 2 \gamma$. The locus of a line that is right parallel to one of the generators of a cone of order $m$ and left parallel to the others in order is a ruled non-developable surface $S$ of order $2 m$. Any curve drawn on the surface $S$ and everywhere orthogonal to its generators will meet any generator in points whose distance apart is constant for the given surface and proportional to its volume.

\section{F. N. CoLE,}

Secretary.

\section{THE WINTER MEETING OF THE CHICAGO SECTION.}

THE twenty-sixth regular meeting of the Chicago Section of the American Mathematical Sucietry was held at the University of Chicago, on Friday and Saturday, December 31, 1909-January 1, 1910. Professor G. A. Miller, Chairman of the Section, presided at all of the sessions except at the opening on Friday morning, when Professor E. B. Van Vleck, VicePresident of the Society, occupied the Chair. The attendance at the various sessions included sixty-one persons among whom were the following forty-seven members of the Society :

Professor C. H. Ashton, Mr. W. H. Bates, Professor G. A. Bliss, Professor Oskar Bolza, Professor J.W. Bradshaw, Professor W. H. Bussey, Dr. Thomas Buck, Professor D. F. Campbell, Professor D. R. Curtiss, Professor J. F. Downey, Dr. Arnold Dresden, Mr. E. B. Escott, Mr. Meyer Gaba, Professor E. D. Grant, Mr. T. H. Hildebrandt, Professor F. H. Hodge, Professor T. F. Holgate, Professor Kurt Laves, Dr. A. C. Lunn, Mr. E. J. Miles, Dr. W. D. MacMillan, Dr. H. F. MacNeish, Professor G. A. Miller, Professor E. H. Moore, Professor C. N. Moore, Dr. R. L. Moore, Professor J. C. Morehead, Professor F. R. Moulton, Professor Alexander Pell, Mrs. Anna J. Pell, Miss Ida M. Schottenfels, Professor G. A. Scott, Mr. A. R. Schweitzer, Professor J. B. Shaw, Professor C. H. Sisam, Professor E. B. Skinner, Professor H. E. Slaught, Professor A. W. Smith, Professor A. L. Underhill, Professor E. B. Van 